\title{
Asthma health services utilisation before, during and after pregnancy: a population- based cohort study
}

\author{
Teresa To ${ }^{1,2,3}$, Laura Y. Feldman ${ }^{1}$, Jingqin Zhu ${ }^{1,2}$ and Andrea S. Gershon ${ }^{1,2,3,4}$ \\ Affiliations: ${ }^{1}$ Child Health Evaluative Sciences, The Hospital for Sick Children, Toronto, ON, Canada. ${ }^{2}$ Institute \\ for Clinical Evaluative Sciences, Toronto, ON, Canada. ${ }^{3}$ Dalla Lana School of Public Health, University of \\ Toronto, Toronto, ON, Canada. ${ }^{4}$ Sunnybrook Health Sciences Centre, Toronto, ON, Canada.
}

Correspondence: Teresa To, Child Health Evaluative Sciences, The Hospital for Sick Children, 686 Bay Street, Toronto, ON, M5G 0A4, Canada. E-mail: teresa.todasickkids.ca

@ERSpublications

Females in Ontario, Canada have increased hospitalisations and reduced primary care visits for asthma during pregnancy http://ow.ly/9yqi30iUJQM

Cite this article as: To T, Feldman LY, Zhu J, et al. Asthma health services utilisation before, during and after pregnancy: a population-based cohort study. Eur Respir J 2018; 51: 1800209 [https://doi.org/10.1183/ 13993003.00209-2018].

ABSTRACT During pregnancy, females with asthma may be at higher risk of exacerbation. The objective of this study was to determine whether females with asthma in Ontario, Canada have increased health services utilisation (HSU) during pregnancy.

Rates of asthma-specific, asthma-related and non-pregnancy-related HSU were calculated in a population-based cohort of pregnant females with asthma. Poisson regression with repeated measures was used to determine adjusted rate ratios and 95\% confidence intervals of HSU during and 1 year after pregnancy, compared to the year before pregnancy.

The cohort consisted of 103976 pregnant females with asthma. Compared to the year prior to pregnancy, hospitalisation rates per 100 person-months during pregnancy increased $30 \%$ for asthma (from 0.016 to 0.020 ), $24 \%$ for asthma-related conditions (from 0.012 to 0.015 ) and decreased $37 \%$ for nonpregnancy-related conditions (from 0.24 to 0.15 ). Emergency department visits for asthma and asthmarelated conditions did not increase significantly during pregnancy. During pregnancy, physician office visits decreased $19 \%$ for asthma (from 2.20 to 1.79), 10\% for asthma-related conditions (from 9.44 to 8.47 ) and increased $74 \%$ for non-pregnancy-related conditions (from 56.4 to 98.2).

Hospitalisations for asthma and asthma-related conditions increased during pregnancy, demonstrating that the overall increase in non-pregnancy-related physician office visits may not meet the primary care needs of pregnant females with asthma. 


\section{Introduction}

Several prospective studies of pregnant females with asthma have demonstrated that, during pregnancy, asthma tends to improve in one-third of females, stays the same in one-third and worsens in one-third $[1,2]$.

The prognosis of asthma during pregnancy has been shown to be affected by provider and patient factors. An Australian survey demonstrated that many general practitioners involved in the primary care of pregnant females decreased patients' asthma medications during pregnancy even when asthma was well-controlled by current therapy, despite reporting a good knowledge of asthma [3]. Healthcare providers in North America have been shown to delay or reduce prescription of systemic corticosteroids to pregnant females during asthma exacerbations treated in acute care settings, compared to non-pregnant females [4-7]. Many pregnant females themselves have been reported to reduce or discontinue asthma controller medications during pregnancy, due to concern about the effect of asthma medications on the fetus $[8,9]$. Other factors that may affect the prognosis of asthma during pregnancy include pre-pregnancy severity of asthma, viral infections, allergic rhinitis and smoking [2, 10-13].

Deviation from asthma guidelines during pregnancy may place pregnant females at higher risk of asthma exacerbations, thus leading to avoidable maternal and possibly fetal morbidity, and an increased burden on the healthcare system. A systematic review of 33 published studies did not find any conclusive links between preventive asthma medications and adverse pregnancy outcomes [14]. The authors of the study recommended that healthcare providers follow asthma guidelines produced by professional organisations such as the Global Initiative for Asthma (GINA), which emphasises the importance of actively treating asthma during pregnancy and not stepping down medications even if asthma is well-controlled [15]. Furthermore, it has been demonstrated that the use of asthma medications below levels recommended by GINA is associated with more severe asthma during pregnancy [13].

In order to understand the unique healthcare needs of pregnant females with asthma and the impact that these needs have on the healthcare system, it is important to describe the patterns and risk factors of healthcare utilisation of this vulnerable population. The objective of this study was to determine whether females with asthma in Ontario, Canada have increased health services utilisation (HSU) during pregnancy, compared to before and after pregnancy, and to identify individual as well as community level risk factors for increased HSU.

\section{Methods}

\section{Study design}

In this population-based cohort study, pregnant females with asthma living in Ontario, Canada were followed using health administrative databases to determine HSU for asthma, asthma-related conditions and non-pregnancy-related conditions. HSU was evaluated at three time points: in the year before pregnancy, during pregnancy and in the first year post-delivery.

Approval to conduct this study was obtained from the Institute for Clinical Evaluative Sciences (ICES) and the research ethics board at the Hospital for Sick Children (Toronto, Canada).

\section{Study population}

The study population consisted of pregnant females with pre-existing asthma aged $\geqslant 19$ years living in Ontario, Canada. Females with asthma were identified from the Ontario Asthma Surveillance Information System (OASIS; http://lab.research.sickkids.ca/oasis/), which includes all individuals covered by Ontario's universal healthcare system who meet the following definition of asthma: one or more hospitalisations for asthma or two or more outpatient claims for asthma in two consecutive years [16]. Females were included if they delivered between April 1, 2005 and March 31, 2015 and if their pregnancy had gestational age between 37 and 42 weeks (inclusive) and did not result in termination, stillbirth or multiple births. Females were further excluded if, during the study period (i.e. within the year prior to and first year post-delivery), they had another delivery or had incomplete health insurance coverage or data. Fewer than six females had congestive heart failure; in order to protect individual identities, they were removed during the initial screening process.

If females had more than one delivery during the study period, only the first delivery that met inclusion/ exclusion criteria was used in this study.

\section{Data sources}

All study data were captured in six large, population-based health administrative databases housed at ICES (online supplementary eTable 1). Individuals in the study cohort were linked across these databases using their encrypted, unique health card number, given to every Ontario resident covered by Ontario's universal healthcare system. 


\section{Exposure and outcome measures}

Exposure

The three exposure times investigated were the year prior to pregnancy, during pregnancy and the first year post-delivery. These dates are calculated using the mother's gestation weeks at delivery and the newborn's date of birth, as recorded in health administrative databases.

\section{Outcomes}

Asthma

HSU was captured using the diagnostic code 493 (International Classification of Diseases (ICD)-9) or J45/ J46 (ICD-10) (online supplementary eTable 2).

\section{Asthma-related conditions}

HSU was captured using diagnostic codes for acute respiratory infections, pneumonia, influenza, chronic obstructive pulmonary disease (COPD), atopic dermatitis, gastro-oesophageal reflux disorder, heartburn and allergic contact dermatitis (online supplementary eTable 2).

\section{Non-pregnancy-related conditions}

HSU was captured using any diagnostic code except for the following ICD chapter headings: complications of pregnancy, childbirth and the puerperium and certain conditions originating in the perinatal period (online supplementary eTable 2). Therefore, non-pregnancy-related conditions included asthma and asthma-related conditions.

Three forms of HSU were captured for each group of conditions: hospitalisations, emergency department visits and physician office visits. For hospitalisations and emergency department visits, only primary diagnostic codes were considered. For physician office visits, only one diagnosis code is available and is used per patient visit.

\section{Statistical analysis}

Counts of HSU were tabulated for the three time periods of the study, and rates per 100 person-months were calculated. This approach ensures that rates can be compared between the three time periods, which vary in duration from approximately nine to 12 months.

Poisson regression with repeated measures using generalised estimating equations was used to generate rate ratios (RR) and 95\% confidence intervals for the effect of each period of pregnancy on HSU (reference period: the year prior to pregnancy). Separate models were run for the three groups of conditions (asthma, asthma-related conditions and non-pregnancy-related conditions) and each type of HSU. HSU was modelled as rates, using the number of days in each time period as an offset. To examine if HSU differed by maternal age at delivery, duration of maternal asthma, material deprivation, rural/urban residence and the presence of certain comorbidities, these variables were included as covariates in multivariable regressions. The duration of maternal asthma was calculated as the number of years since each woman was first included in OASIS. Material deprivation, a measure of socioeconomic status (SES), was measured using the Ontario Marginalization Index [17]. The following comorbidities were considered at each of the three exposure periods: diabetes, cardiovascular disease, COPD and cancer (defined in online supplementary eTable 3).

\section{Results}

\section{Descriptive data}

The study cohort consisted of 103976 pregnant females with asthma with one singleton delivery each (figure 1). Pregnant females with asthma were aged mean \pm SD $28.9 \pm 5.5$ years at delivery (table 1 ). They were first included in OASIS at an average age of 16.4 \pm 8.2 years and had an average duration of asthma of $12.5 \pm 5.5$ years. $22.8 \%$ of the females were in the highest quintile of material deprivation (most deprived), whereas $23.0 \%$ were in the lowest quintile, and $11.2 \%$ lived in rural areas. The most common comorbidity was cardiovascular disease $(8.7 \%$ in the year prior to pregnancy) followed by diabetes $(1.7 \%)$.

\section{Rates of HSU}

Compared to the year prior to pregnancy, hospitalisations for asthma and asthma-related conditions increased during pregnancy, after which they dropped markedly in the first year post-delivery (figure 2). Emergency department visits for asthma and asthma-related conditions were stable during pregnancy, after which they dropped markedly in the first year post-delivery. In contrast, acute care visits (hospitalisations and emergency department visits) decreased during pregnancy for non-pregnancy-related conditions, after which hospitalisations rebounded to pre-pregnancy levels and emergency department visits continued to decrease. Physician office visits for asthma and asthma-related conditions decreased during pregnancy and 
FIGURE 1 Flow diagram of the assembly of the study cohort.

$n=118738$

Females with asthma aged $\geqslant 19$ years living in Ontario, Canada with delivery between April 1, 2005 and March 31, 2015 after their asthma diagnosis

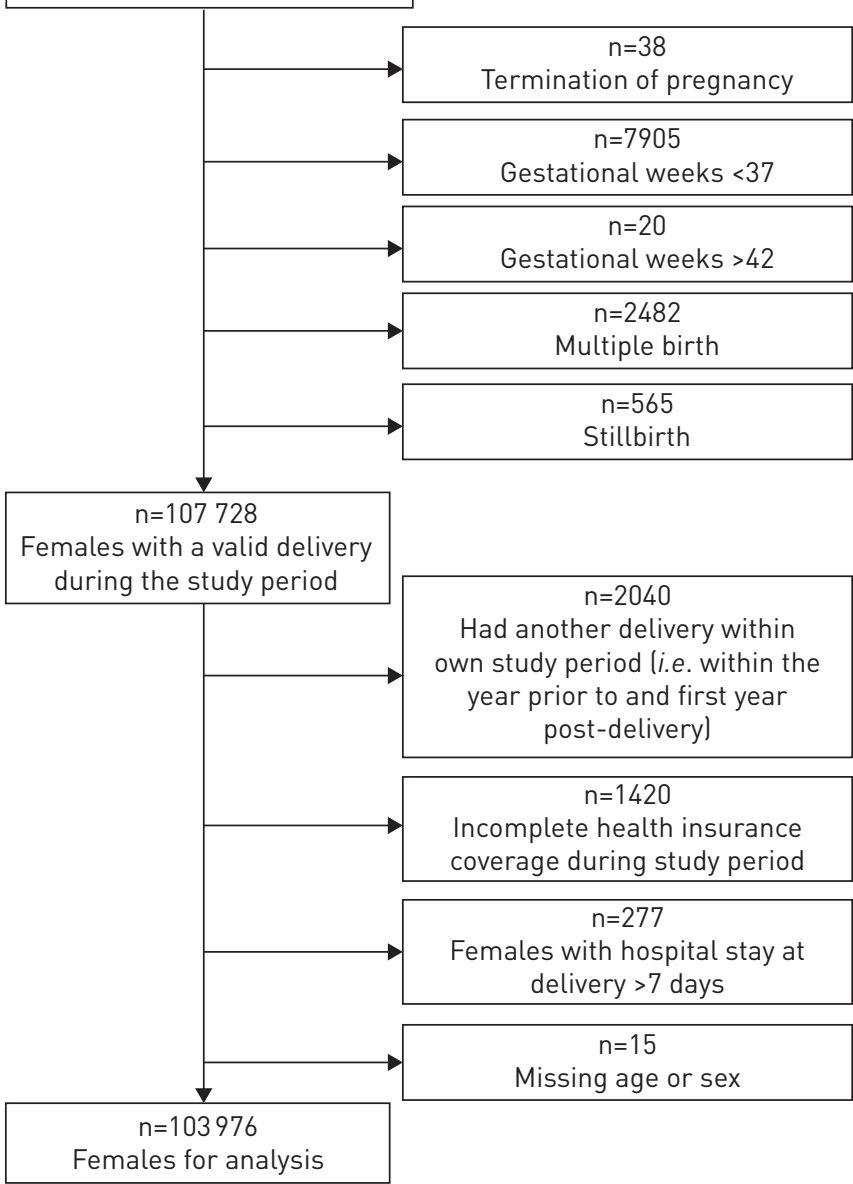

in the first year post-delivery, whereas they increased during pregnancy for non-pregnancy-related conditions (figure 2).

Rates further broken down by trimester of pregnancy are shown in online supplementary eFigure 1. While hospitalisations increased steadily across all trimesters of pregnancy for asthma, they were only increased in the second and third trimesters for asthma-related conditions. Emergency department visits for asthma and asthma-related conditions were highest in the second trimester, and emergency department visits for non-pregnancy-related conditions peaked in the first trimester. The increase in physician office visits for non-pregnancy-related conditions during pregnancy was most pronounced in the third trimester.

\section{Multivariable analysis}

Figure 3 displays RR (95\% CI) for HSU during pregnancy and in the first year post-delivery (reference period: the year prior to pregnancy). In addition, covariate effects are displayed.

\section{Hospitalisations}

Multiple regression analyses could not be conducted for asthma or asthma-related hospitalisations, due to too few outcomes occurring for these conditions. Compared to the year prior to pregnancy, hospitalisations for non-pregnancy-related conditions decreased 37\% during pregnancy $(0.63,0.59-0.68)$, rebounding to baseline levels in the first year post-delivery $(0.99,0.94-1.05)$.

\section{Emergency department visits}

Compared to the year prior to pregnancy, emergency department visits increased $6 \%$ for asthma and remained the same for asthma-related conditions during pregnancy $(1.06,1.00-1.12$ and $0.99,0.96-1.03$, 
TABLE 1 Characteristics of pregnant females with asthma who delivered between April 1, 2005 and March 31, 2015

\begin{tabular}{lr} 
Subjects & 103976 \\
Mother's age at discharge of the delivery & $28.9 \pm 5.5$ \\
Duration of asthma years & $12.5 \pm 5.5$ \\
Mother's age when first captured in provincial asthma surveillance system & $16.4 \pm 8.2$ \\
Gestation at delivery weeks & $39.2 \pm 1.2$ \\
Deaths & $241(0.2)$ \\
Deprivation quintile & \\
$\quad 1$ (least deprived) & $23611(23.0)$ \\
2 & $18552(18.1)$ \\
3 & $18609(18.1)$ \\
4 & $18428(18.0)$ \\
5 (most deprived) & $23392(22.8)$ \\
Rurality & \\
Urban & $92274(88.8)$ \\
Rural & $11674(11.2)$ \\
Comorbidities & \\
Cardiovascular disease & \\
$\quad$ Year prior to pregnancy & $9021(8.7)$ \\
$\quad$ During pregnancy & $9709(9.3)$ \\
$\quad$ Year post-delivery & $10508(10.1)$ \\
Diabetes & \\
$\quad$ Year prior to pregnancy & $1778(1.7)$ \\
During pregnancy & $2032(2.0)$ \\
Year post-delivery & $2331(2.2)$ \\
Cancer & \\
Year prior to pregnancy & $540(0.5)$ \\
During pregnancy & $595(0.6)$ \\
Year post-delivery & $621(0.6)$ \\
COPD & \\
$\quad$ Year prior to pregnancy & $206(0.2)$ \\
During pregnancy & $308(0.3)$ \\
$\quad$ Year post-delivery & $373(0.4)$ \\
& \\
\hline
\end{tabular}

Data are presented as $\mathrm{n}$, mean \pm SD or $\mathrm{n}(\%)$. Percentages are adjusted for missingness. COPD: chronic obstructive pulmonary disease. ${ }^{\#}$ : comorbidities were tabulated at the beginning of each time period of pregnancy. Categories are not mutually exclusive.

respectively) then decreased by $\sim 40 \%$ in the first year post-delivery $(0.53,0.49-0.57$ and $0.67,0.65-0.70$, respectively). Emergency department visits for non-pregnancy-related conditions decreased $8 \%$ during pregnancy and $27 \%$ in the first year post-delivery $(0.92,0.91-0.94$ and $0.74,0.72-0.74$, respectively).

\section{Physician office visits}

Compared to the year prior to pregnancy, physician office visits decreased $19 \%$ for asthma and $10 \%$ for asthma-related conditions during pregnancy $(0.81,0.79-0.83$ and $0.90,0.88-0.91$, respectively) and $28 \%$ and $11 \%$ in the first year post-delivery $(0.72,0.71-0.74$ and $0.89,0.88-0.90$, respectively). Physician office visits for non-pregnancy-related conditions increased $74 \%$ during pregnancy $(1.74,1.72-1.75)$ and nearly returned to baseline levels in the first year post-delivery $(1.02,1.01-1.03)$.

\section{Covariate effects}

Increased material deprivation was generally associated with increased HSU. Compared to being in the lowest (least deprived) quintile of material deprivation, being in the highest quintile was associated with double the emergency department visits and $22 \%$ more physician office visits for asthma $(1.95,1.74-2.20$ and 1.22, 1.16-1.29, respectively). Similarly, being in the highest quintile of material deprivation was associated with a $41 \%$ higher hospitalisation rate and a $73 \%$ higher emergency department visit rate for non-pregnancy-related conditions (1.41, 1.28-1.55 and 1.73, 1.67-1.80, respectively), but was not as strongly associated with physician office visits $(1.02,1.00-1.03)$.

Females in rural areas experienced a higher rate of hospitalisations and emergency department visits and a lower rate of physician office visits, compared to females in urban areas. For example, females in rural 


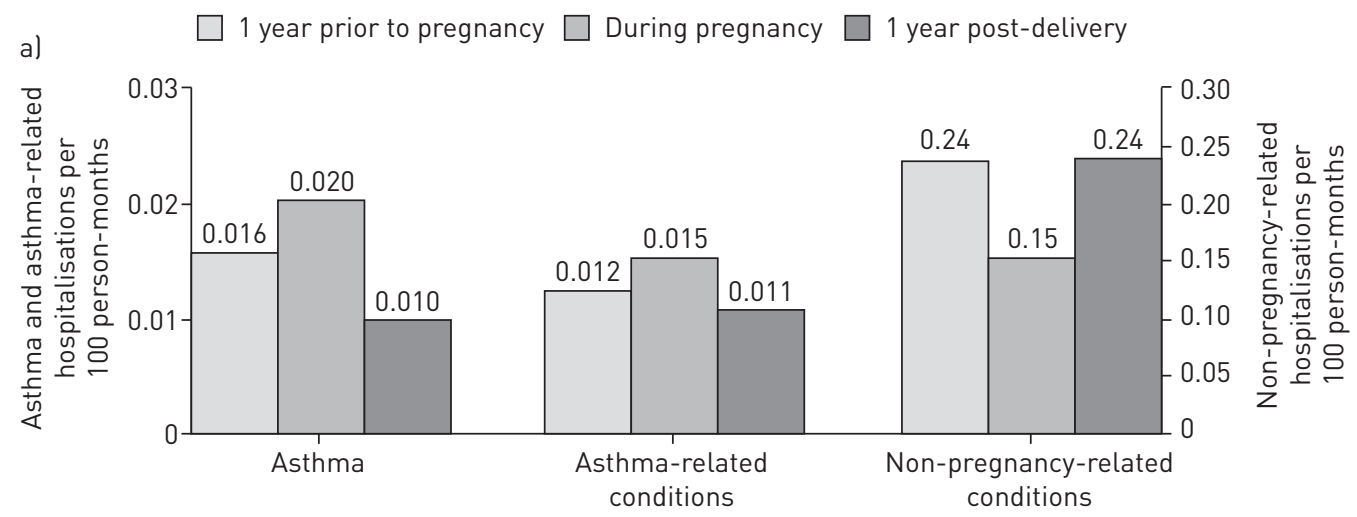

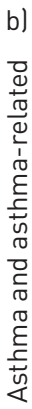
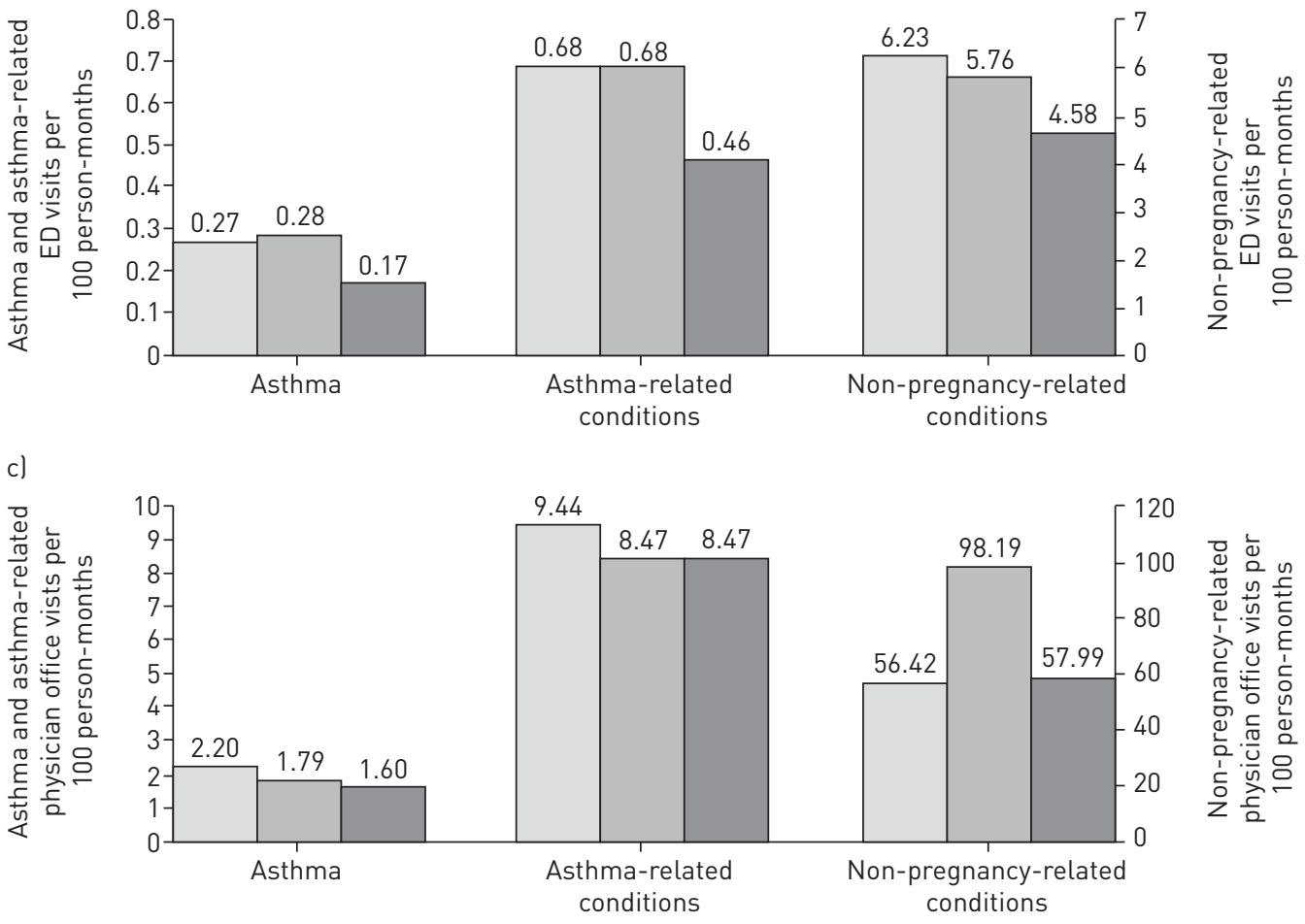

FIGURE 2 Rates per 100 person-months of follow-up for a) hospitalisations, b) emergency department (ED) visits and c) physician office visits for asthma, asthma-related conditions and non-pregnancy-related conditions during the three time periods in this study.

areas experienced an $85 \%$ higher emergency department visit rate and $32 \%$ lower physician office visit rate for asthma $(1.85,1.68-2.04$ and $0.68,0.64-0.73$, respectively).

Comorbidities were generally associated with increased HSU. COPD was the comorbidity associated with some of the greatest increases in HSU, including emergency department visits for asthma, asthma-related conditions and non-pregnancy-related conditions $(3.87,2.08-7.17$; 2.39, 1.51-3.76; and 2.05, 1.63-2.57, respectively). Diabetes was associated with the greatest increases in physician office visits for non-pregnancy-related conditions $(1.50,1.46-1.54)$.

\section{Discussion}

Our study used a population-based cohort of pregnant females with asthma to compare patterns of HSU before, during and after pregnancy. We reported a significant increase in hospitalisations during pregnancy for asthma and asthma-related conditions. In addition, we found that physician office visits for non-pregnancy-related conditions were increased during pregnancy, whereas physician office visits for asthma and asthma-related conditions decreased. Higher levels of material deprivation, rural residence and the presence of comorbidities were associated with higher rates of HSU among pregnant females with 
a) Hospitalisations

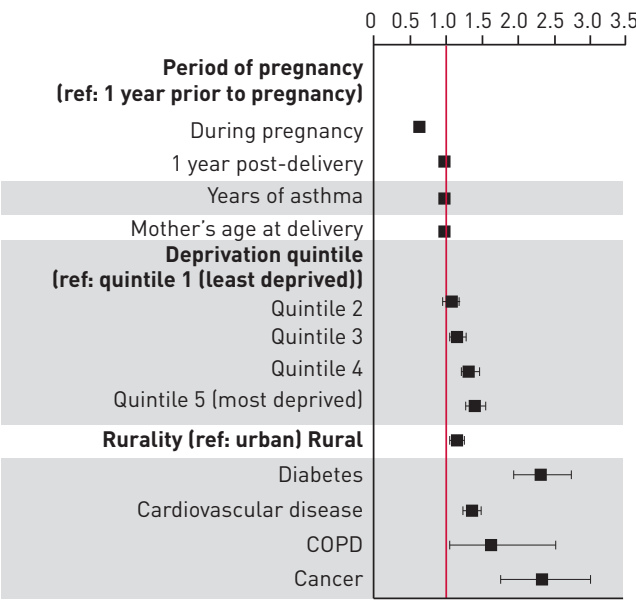

b) ED visits

All non-pregnancy-

related conditions

$\mathrm{RR}(95 \% \mathrm{Cl})$

$0 \quad 0.51 .01 .52 .0253 .03 .5$

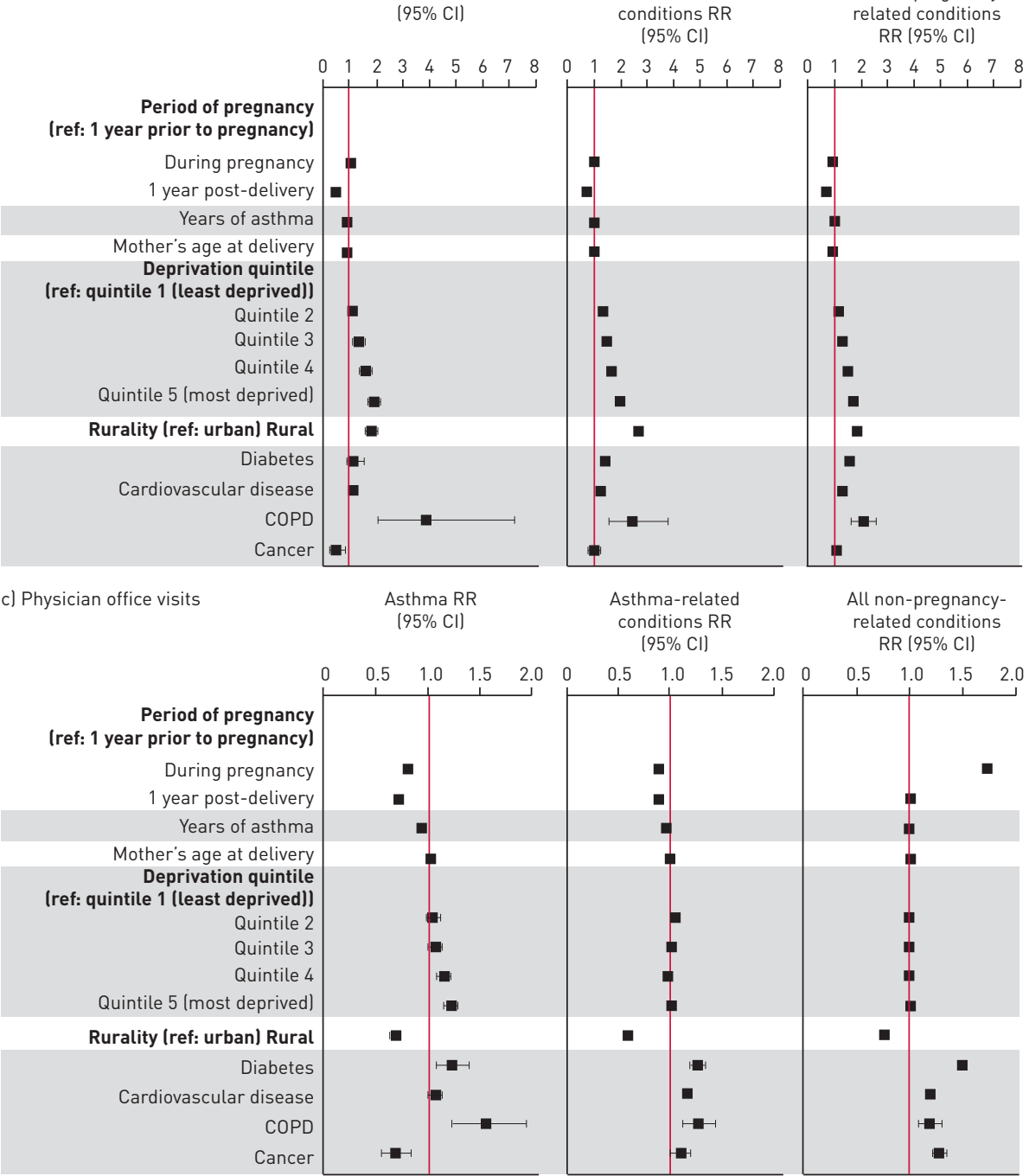

FIGURE 3 Rate ratios (RR) and 95\% confidence intervals from Poisson regression with repeated measures describing exposure and covariate effects on a) hospitalisations, b) emergency department (ED) visits and c) physician office visits for asthma, asthma-related conditions and non-pregnancy-related conditions. Multiple regression analyses could not be conducted for asthma or asthma-related hospitalisations, due to too few outcomes occurring for these conditions. COPD: chronic obstructive pulmonary disease. 
asthma. The increase in hospitalisations for asthma and asthma-related conditions during pregnancy suggests a role for asthma-focused primary care during pregnancy.

\section{Interpretation of findings in broader context of existing literature}

KIM et al. [18] examined asthma HSU in 3357 pregnant and 50355 non-pregnant females using the Korean National Health Insurance claim database. They examined patterns of HSU in the year prior to pregnancy, during pregnancy and in the first and second years after delivery. Crude rates of asthma HSU were higher in the study by KIM et al. than in our study, probably because their inclusion criteria included prior asthma HSU and asthma-related medications or tests during the study period; therefore, their cohort would have been more likely to have "active" disease than the females in our study. However, the overall patterns of HSU were very similar to our study. As in our study, they showed that hospitalisations for asthma increased during pregnancy, whereas outpatient visits were significantly lower. In contrast to our findings, KIM et al. showed that emergency department visits declined during pregnancy and in the first year post-delivery. However, increases in hospitalisations and decreases in the number of emergency department visits for asthma during pregnancy were not statistically significant in their study, in which only a small number of acute care visits were seen.

The observed peak in emergency department visits for asthma during the second trimester of pregnancy is consistent with prospective cohort studies that have found asthma exacerbations to be most frequent between gestational weeks 17 and 24 [19, 20]. In our study, hospitalisations for asthma, which accounted for a minority $(\sim 10 \%)$ of acute care visits for asthma, peaked in the third trimester of pregnancy. This is consistent with the study by KIM et al. [18] and may reflect an increased tendency toward hospitalisation among pregnant females with asthma presenting to the emergency department in the third trimester with respiratory complaints.

The association between material deprivation and HSU observed in our study is consistent with both Canadian and international literature. In Canada, low SES has previously been reported to be associated with increased healthcare utilisation for asthma and other chronic health conditions, as well as generally higher healthcare utilisation [21-26]. Some of this association may be explained by differing health needs across levels of SES [27]. International studies have also supported an association between lower SES and increased asthma HSU [28, 29].

In Ontario, Canada, there is a low availability of primary care physician services in some rural areas, particularly in northern areas of the province; this is probably the reason that physician office visits are generally lower for females with rural residence, while hospitalisations and emergency department visits are higher [30].

The increase in hospitalisations for asthma and asthma-related conditions during pregnancy could be explained by a well-documented decrease in adherence to asthma medications during pregnancy. ENRIQUEZ et al. [9] analysed prescriptions filled by $>8000$ pregnant females with asthma who were enrolled in Tennessee Medicaid between 1995 and 2001. Although their use of health administrative data did not allow them to comment on reasons for the patterns they observed, ENRIQuez et al. demonstrated that pregnant females filled $22.9 \%$ fewer prescriptions for inhaled corticosteroids (ICS), $13.2 \%$ fewer prescriptions for short-acting $\beta$-agonists and $54.3 \%$ fewer prescriptions for rescue oral corticosteroids from 5 to 13 weeks after the last menstrual period. Using health administrative databases to analyse 4920 pregnancies among females with asthma in Québec, Canada, BLAIs et al. [31] found that $48.5 \%$ of pregnancies were in females who discontinued or reduced their ICS use during pregnancy. Interestingly, BLAIS et al. found that females who discontinued ICS experienced fewer asthma exacerbations, while females who increased their use of ICS experienced more, probably reflecting residual confounding by asthma severity.

\section{Strengths and limitations}

Strengths of this study include the use of large, population-based databases covering the entire province of Ontario. The linkage of these large, population-based databases allowed us to assemble a longitudinal cohort to observe HSU before, during and after pregnancy for $>10$ years. The use of prospectively collected, population-based data reduces the risk of recall and selection bias and increases the generalisability of our results.

Limitations of this study include a lack of clinical variables, making it impossible for us to control for or stratify analyses by pre-pregnancy asthma severity. However, in our cohort study, females assessed for outcomes during pregnancy were the same females assessed for outcomes during the pre-pregnancy period. Therefore, the increase in acute care visits for asthma and asthma-related conditions seen during pregnancy cannot be due to a greater proportion of people with severe asthma being present in the study 
during this time period. The inclusion of live, singleton births at 37-42 weeks gestation resulted in the exclusion of preterm births, which are associated with asthma exacerbation [32]. Therefore, the magnitude of the association between pregnancy and acute asthma HSU is probably greater than that reported in our study. The use of health administrative data does not allow us to determine whether patterns of HSU changed due to disease worsening, or due to a change in health behaviours such as greater concern for the health of pregnant females. However, we observed a decrease in acute care visits for non-pregnancy-related conditions, suggesting that the increase in hospitalisations we observed for asthma and asthma-related conditions was not merely a signal of overall changed health behaviours. Lastly, the lack of prescription data for those in Ontario aged $<65$ years makes us unable to examine medication prescriptions as potential mechanisms underlying the association between periods of pregnancy and HSU.

\section{Conclusions}

We conducted a population-based study of pregnant females with asthma and found an increase in hospitalisations and a decrease in physician office visits during pregnancy for asthma and asthma-related conditions, suggesting less preventative and more reactive treatment. The observed increase in physician office visits for non-pregnancy-related conditions during pregnancy does not appear to meet the need for asthma-focused primary care during pregnancy. Since avoidance of hospitalisations for asthma is highly important during pregnancy, these findings point to a possible need for more preventive, focused physician office visits to ensure asthma is under control.

Support statement: The Ontario Asthma Surveillance Information System (OASIS) is funded by the Ontario Ministry of Health and Long-term Care (MOHLTC). Data are provided by the Institute for Clinical Evaluative Sciences (ICES), which is funded by an annual grant from the MOHLTC. The opinions, results and conclusions reported in this paper are those of the authors and are independent from the funding sources. No endorsement by ICES or the Ontario MOHLTC is intended or should be inferred. Parts of this material are based on data and information compiled and provided by the Canadian Institute for Health Information (CIHI). However, the analyses, conclusions, opinions and statements expressed herein are those of the authors, and not necessarily those of CIHI. The authors have no other relevant sources of funding to declare. A.S. Gershon holds a New Investigator Career Award from the Canadian Institutes of Health Research (CIHR) and is the recipient of a Physician Services Incorporated (PSI) Foundation Fellowship for Translational Health Research. Funding information for this article has been deposited with the Crossref Funder Registry.

Conflict of interest: None declared.

\section{References}

1 Schatz M, Harden K, Forsythe A, et al. The course of asthma during pregnancy, post partum, and with successive pregnancies: a prospective analysis. J Allergy Clin Immunol 1988; 81: 509-517.

2 Schatz M, Dombrowski MP, Wise R, et al. Asthma morbidity during pregnancy can be predicted by severity classification. J Allergy Clin Immunol 2003; 112: 283-288.

3 Lim AS, Stewart K, Abramson MJ, et al. Management of asthma in pregnant women by general practitioners: a cross sectional survey. BMC Fam Pract 2011; 12: 121

4 Cossette B, Beauchesne M-F, Forget A, et al. Systemic corticosteroids for the treatment of asthma exacerbations during and outside of pregnancy in an acute-care setting. Respir Med 2014; 108: 1260-1267.

5 Cydulka RK, Emerman CL, Schreiber D, et al. Acute asthma among pregnant women presenting to the emergency department. Am J Respir Crit Care Med 1999; 160: 887-892.

6 Hasegawa K, Cydulka RK, Sullivan AF, et al. Improved management of acute asthma among pregnant women presenting to the ED. Chest 2015; 147: 406-414.

7 McCallister JW, Benninger CG, Frey HA, et al. Pregnancy related treatment disparities of acute asthma exacerbations in the emergency department. Respir Med 2011; 105: 1434-1440.

8 Lim AS, Stewart K, Abramson MJ, et al. Asthma during pregnancy: the experiences, concerns and views of pregnant women with asthma. J Asthma 2012; 49: 474-479.

9 Enriquez R, Wu P, Griffin MR, et al. Cessation of asthma medication in early pregnancy. Am J Obstet Gynecol 2006; 195: 149-153.

10 Murphy VE, Clifton VL, Gibson PG. Asthma exacerbations during pregnancy: incidence and association with adverse pregnancy outcomes. Thorax 2006; 61: 169-176.

11 Ali Z, Ulrik CS. Incidence and risk factors for exacerbations of asthma during pregnancy. J Asthma Allergy 2013; 6: 53-60.

12 Kircher S, Schatz M, Long L. Variables affecting asthma course during pregnancy. Ann Allergy Asthma Immunol 2002; 89: 463-466.

13 Belanger K, Hellenbrand ME, Holford TR, et al. Effect of pregnancy on maternal asthma symptoms and medication use. Obstet Gynecol 2010; 115: 559-567.

14 Lim A, Stewart K, König K, et al. Systematic review of the safety of regular preventive asthma medications during pregnancy. Ann Pharmacother 2011; 45: 931-945.

15 Global Initiative for Asthma (GINA). 2018 GINA Report, Global Strategy for Asthma Management and Prevention. www.ginasthma.org/download/836

16 Gershon AS, Wang C, Guan J, et al. Identifying patients with physician-diagnosed asthma in health administrative databases. Can Respir J 2009; 16: 183-188. 
17 Matheson FI, Dunn JR, Smith KLW, et al. ON-Marg Ontario Marginalization Index User Guide. Toronto, Centre for Research on Inner City Health, 2012.

18 Kim S, Kim J, Park SY, et al. Effect of pregnancy in asthma on health care use and perinatal outcomes. J Allergy Clin Immunol 2015; 136: 1215-1223.

19 Murphy VE, Gibson P, Talbot PI, et al. Severe asthma exacerbations during pregnancy. Obstet Gynecol 2005; 106: 1046-1054.

20 Stenius-Aarniala BS, Hedman J, Teramo KA. Acute asthma during pregnancy. Thorax 1996; 51: 411-414.

21 Bacon SL, Bouchard A, Loucks EB, et al. Individual-level socioeconomic status is associated with worse asthma morbidity in patients with asthma. Respir Res 2009; 10: 125.

22 Lasser KE, Himmelstein DU, Woolhandler S. Access to care, health status, and health disparities in the United States and Canada: results of a cross-national population-based survey. Am J Public Health 2006; 96: 1300-1307.

23 Booth GL, Hux JE. Relationship between avoidable hospitalizations for diabetes mellitus and income level. Arch Intern Med 2003; 163: 101-106.

24 Roos LL, Walld R, Uhanova J, et al. Physician visits, hospitalizations, and socioeconomic status: ambulatory care sensitive conditions in a Canadian setting. Health Serv Res 2005; 40: 1167-1185.

25 Fitzpatrick T, Rosella LC, Calzavara A, et al. Looking beyond income and education: socioeconomic status gradients among future high-cost users of health care. Am J Prev Med 2015; 49: 161-171.

26 Rosella LC, Fitzpatrick T, Wodchis WP, et al. High-cost health care users in Ontario, Canada: demographic, socio-economic, and health status characteristics. BMC Health Serv Res 2014; 14: 532.

27 Blackwell DL, Martinez ME, Gentleman JF, et al. Socioeconomic status and utilization of health care services in Canada and the United States: findings from a binational health survey. Med Care 2009; 47: 1136-1146.

28 Weiss KB, Gergen PJ, Crain EF. Inner-city asthma: the epidemiology of an emerging US public health concern. Chest 1992; 101: 362S-367S.

29 Watson JP, Cowen P, Lewis RA. The relationship between asthma admission rates, routes of admission, and socioeconomic deprivation. Eur Respir J 1996; 9: 2087-2093.

30 Hay C, Pacey M, Bains N, et al. Understanding the unattached population in Ontario: evidence from the Primary Care Access Survey (PCAS). Healthc Policy 2010; 6: 33-47.

31 Blais L, Firoozi F, Kettani F-Z, et al. Relationship between changes in inhaled corticosteroid use and markers of uncontrolled asthma during pregnancy. Pharmacotherapy 2012; 32: 202-209.

32 Murphy VE, Namazy JA, Powell H, et al. A meta-analysis of adverse perinatal outcomes in women with asthma. BJOG 2011; 118: 1314-1323. 